\title{
Berberine attenuates hepatic oxidative stress in rats with non-alcoholic fatty liver disease via the Nrf2/ARE signalling pathway
}

\author{
YUANJUN DENG ${ }^{*}$, KAIRUI TANG**, RUNSEN CHEN, HUAN NIE, SHU LIANG, \\ JINWEN ZHANG, YUPEI ZHANG and QINHE YANG
}

School of Traditional Chinese Medicine, Jinan University, Guangzhou, Guangdong 510632, P.R. China

Received June 30, 2018; Accepted December 21, 2018

DOI: $10.3892 /$ etm.2019.7208

\begin{abstract}
The present study investigated the effects of berberine (BBR) on hepatic oxidative stress and the nuclear factor erythroid 2-related factor 2/antioxidant response element (Nrf2/ARE) signalling pathway in rats in which non-alcoholic fatty liver disease (NAFLD) was induced by a high-fat diet. Rats were randomly divided into three groups: The normal control (NC), high-fat diet (HFD) and BBR groups. The NC group received a normal diet, while the other two groups were fed a high-fat diet. The rats in the BBR group were also fed BBR (100 mg/kg body weight) daily. A total of 8 weeks later, serum and liver lipid levels were measured. Hepatic histopathological changes were observed with haematoxylin and eosin and Oil Red O staining. Transmission electron microscopy was performed to observe the ultrastructural changes of the liver. The levels of superoxide dismutase (SOD), glutathione (GSH) and malondialdehyde (MDA) in the liver were measured. Quantitative polymerase chain reaction and western blotting were performed to investigate the expression of genes in the Nrf2/ARE signalling pathway in the liver. Histopathological
\end{abstract}

Correspondence to: Professor Qinhe Yang or Dr Yupei Zhang, School of Traditional Chinese Medicine, Jinan University, 601 Huangpu Avenue West, Guangzhou, Guangdong 510632, P.R. China

E-mail: tyangqh@jnu.edu.cn

E-mail: zyp6115@jnu.edu.cn

*Contributed equally

Abbreviations: BBR, berberine; GSH, glutathione; HDL-C, high-density lipoprotein cholesterol; HO-1, haeme oxygenase-1; Keap-1, kelch-like epichlorohydrin-associated protein 1; LDL-C, low-density lipoprotein cholesterol; MDA, malondialdehyde; $\mathrm{Nrf2}$, nuclear factor erythroid 2-related factor 2; NQO1, NAD(P)H dehydrogenase [quinone] 1; SOD, superoxide dismutase; TC, total cholesterol; TG, triglyceride

Key words: non-alcoholic fatty liver disease, berberine, nuclear factor erythroid 2-related factor 2, oxidative stress results demonstrated that rats fed a high-fat diet for 8 weeks developed NAFLD, characterized by hepatic steatosis. BBR significantly decreased the body weight and liver weight. BBR markedly reduced hepatic steatosis, and the serum and liver lipid levels. Hepatic SOD and GSH levels were increased, while MDA levels were decreased by BBR co-administered with a high-fat diet. Additionally, the Nrf2/ARE signalling pathway was revealed to be involved in the protective effect of BBR on rats fed a high-fat diet. In conclusion, BBR may alleviate hepatic oxidative stress in rats with NAFLD, which may be partly attributed to the activation of the Nrf2/ARE signalling pathway.

\section{Introduction}

Non-alcoholic fatty liver disease (NAFLD), which is characterized by the presence of hepatic steatosis in the absence of excessive alcohol consumption, is recognized as the typical hepatic manifestation of metabolic syndrome (1). It has become the most common cause of chronic liver disease worldwide, and its prevalence has risen rapidly with the rise of obesity, type 2 diabetes mellitus and metabolic syndrome epidemics $(2,3)$. NAFLD represents a wide spectrum of pathological hepatic changes ranging from simple steatosis to steatohepatitis to different degrees of fibrosis severity (4). The pathogenesis of NAFLD, which is not fully understood, involves several factors, including insulin resistance, alterations of lipid metabolism, mitochondrial dysfunction and oxidative stress (5). Since the mechanistic understanding of and treatment options for NAFLD remain limited, extensive research efforts have been made to develop novel therapeutics for the disease (6).

Oxidative stress serves a pivotal role in the development of NAFLD $(7,8)$. Nuclear factor erythroid 2-related factor 2 (Nrf2), a cytoprotective transcription factor, serves an important role in cellular defence against oxidative stress by interacting with the antioxidant response element (ARE) sequences of antioxidant and cytoprotective genes $(9,10)$. Under normal conditions, Nrf2 is localized to the cytoplasm through its interaction with the actin-bound protein, Kelch-like epichlorohydrin-associated protein 1 (Keap1). When oxidative stress occurs, Nrf2 dissociates from Keap1 and translocates to the nucleus, where 
it binds to ARE sequences, leading to the expression of antioxidant enzymes $(9,10)$. During the process of oxidative stress, activation of the Nrf2/ARE signalling pathway can protect the liver against oxidative stress by inducing the expression of antioxidant enzymes, including haeme oxygenase 1 (HO-1) and NAD(P)H dehydrogenase [quinone] 1 (NQO1) $(11,12)$. For these reasons, the Nrf2/ARE signalling pathway has been regarded as an important therapeutic target for the prevention and treatment of NAFLD (13).

Berberine (BBR) is a type of isoquinoline alkaloid that was originally isolated from the Chinese medicinal herb Rhizoma coptidis, which has been extensively used to treat diabetes mellitus for more than 1,500 years as part of traditional Chinese medicine (14). BBR has been reported to have pharmacological effects in lowering blood glucose (15), reducing blood lipids (16), improving insulin sensitivity (17) and reducing inflammation (18); these effects may contribute to its efficacy in treating NAFLD. Furthermore, accumulating evidence from animal models and clinical trials suggests that BBR may be a potential drug for NAFLD $(19,20)$, but the underlying molecular mechanisms of the effects of BBR in the prevention and treatment of NAFLD remain far from fully elucidated. In the present study, the authors used a NAFLD rat model induced by a high-fat diet to further investigate whether BBR has a beneficial effect on NAFLD through the regulation of the Nrf2/ARE signalling pathway and the amelioration of oxidative stress in the liver.

\section{Materials and methods}

Animals. A total of 24 specific pathogen-free male Sprague-Dawley rats aged 6-7 weeks (220 20 g) were purchased from the Laboratory Animal Research Center of Guangzhou University of Traditional Chinese Medicine (Approval No. SYXK (Yue) 2013-0117; Guangzhou, China). The rats were housed, approximately five per cage, under conditions of controlled temperature $\left(22-26^{\circ} \mathrm{C}\right)$ and humidity (50-60\%), with a 12-h light/dark cycle, and free access to water and the specified diet.

Experimental design. After 1 week of adaptive feeding, the rats were randomly divided into three groups of 8 rats each: The normal control (NC) group, the high-fat diet (HFD) group and the BBR group. The rat model was established according to a method previously reported by our group; however, the duration was adjusted from 12 to 8 weeks (21). The rats in the NC group had free access to a standard diet (11\% kcal as fat, $20 \% \mathrm{kcal}$ as protein and $69 \% \mathrm{kcal}$ as carbohydrate), while those in the HFD group and BBR group were fed a high-fat diet $(30 \% \mathrm{kcal}$ as fat, $19 \% \mathrm{kcal}$ as protein and $51 \% \mathrm{kcal}$ as carbohydrate). The two supplements were purchased from Guangdong Medical Laboratory Animal Center (Guangzhou, China). The rats in the BBR group were given BBR (Mysun Pharmaceutical Co., Ltd., Chifeng, China) at a dose of $100 \mathrm{mg} / \mathrm{kg}$ body weight by gastrogavage each day $(22,23)$, and those in NC and HFD group were given $10 \mathrm{ml} / \mathrm{kg}$ body weight distilled water. The interventions lasted for 8 weeks. At the end of week 8 , all rats were anesthetized by intraperitoneal injection of $2 \%$ pentobarbital $(40 \mathrm{mg} / \mathrm{kg}$ body weight) and blood samples were then collected from the abdominal aorta. The blood samples were centrifuged at $1,500 \times \mathrm{g}$ for $10 \mathrm{~min}$ at $4^{\circ} \mathrm{C}$ and the clear supernatants were collected. The livers were immediately removed and weighed. All rats were treated in accordance with the Guiding Principles for Animal Experiments approved by the Animal Experimental Ethics Committee of Jinan University (Guangzhou, China).

Biochemical analysis. Serum total cholesterol (TC), triglyceride (TG), high-density lipoprotein cholesterol (HDL-C) and low-density lipoprotein cholesterol (LDL-C) were measured using an automatic biochemical analyser (7600-020; Hitachi, Ltd., Tokyo, Japan). The liver tissues were placed into isopropanol for $15 \mathrm{~min}$ at $4^{\circ} \mathrm{C}$. Homogenates were then acquired using a homogenizer (Qiagen $\mathrm{GmbH}$, Hilden, Germany) and centrifuged at $3,000 \mathrm{x}$ g for $10 \mathrm{~min}$ at $4^{\circ} \mathrm{C}$. The clear supernatants were then collected. Liver TC and TG levels were determined using the automatic biochemical analyser, and expressed as a value relative to initial sample wet weight.

Histopathological evaluation. Fresh liver tissues were fixed in $10 \%$ formalin for $24 \mathrm{~h}$ at $4^{\circ} \mathrm{C}$ or frozen in liquid nitrogen for subsequent experiments. Formalin-fixed liver tissues were dehydrated in ethyl alcohol, dealcoholized in xylene, embedded in paraffin, sliced to a thickness of $5 \mu \mathrm{m}$ and then stained with haematoxylin and eosin. At room temperature, liver sections were stained with haematoxylin for $5 \mathrm{~min}$ and eosin for $2 \mathrm{~min}$. Frozen liver tissues were embedded in optimum cutting temperature compound (Sakura Finetek USA Inc., Torrance, CA, USA), sliced at a thickness of $8 \mu \mathrm{m}$ at $-18^{\circ} \mathrm{C}$ and stained with Oil Red $\mathrm{O}$ (cat. no. D027; Nanjing Jiancheng Technology Co., Ltd., Nanjing, China) for $10 \mathrm{~min}$ at room temperature. All liver sections were observed under a light microscope (magnification, x200). The percentage of Oil Red O-stained area was measured using Image-Pro Plus 6.0 (Media Cybernetics, Rockville, MD, USA). For transmission electron microscopy (TEM), liver tissues were fixed with $2.5 \%$ buffered glutaraldehyde solution overnight at $4^{\circ} \mathrm{C}$ and post-fixed with $1 \%$ osmium tetroxide solution for $1 \mathrm{~h}$ at $4^{\circ} \mathrm{C}$, then embedded in Epon812 epoxy resin. Samples were sliced at a thickness of $80 \mathrm{~nm}$ and stained with uranyl acetate for $15 \mathrm{~min}$ and lead citrate for $5 \mathrm{~min}$ at room temperature. Images were acquired using a transmission electron microscope (magnification, x3,900).

Measurement of oxidative stress markers in the liver. Liver tissues were homogenized in PBS at $4^{\circ} \mathrm{C}$. The homogenates were then centrifuged at $3,000 \mathrm{x}$ g for $10 \mathrm{~min}$ at $4^{\circ} \mathrm{C}$ and the supernatants were collected for subsequent examinations. The protein concentration of supernatants was measured using a bicinchoninic acid assay (BCA) protein assay kit (Nanjing KeyGen Biotech Co., Ltd., Nanjing, China). The levels of superoxide dismutase (SOD; cat. no. A001-3), glutathione (GSH; cat. no. A006-2) and malondialdehyde (MDA; cat. no. A003-1) were measured using corresponding commercial kits (Nanjing Jiancheng Technology Co., Ltd.) following the manufacturer's protocol.

Reverse transcription-quantitative polymerase chain reaction (RT-qPCR) analysis. Total RNA was extracted from liver tissues using RNAiso Plus and cDNA was synthesized 
Table I. Primer sequences for reverse transcription-quantification polymerase chain reaction.

\begin{tabular}{ll}
\hline Gene & \multicolumn{1}{c}{ Primer sequence (5'-3') } \\
\hline GAPDH & Forward: CAACGGGAAACCCATCACCA \\
& Reverse: ACGCCAGTAGACTCCACGACAT \\
Nrf2 & Forward: CTCCTTAGACTCAAATCCCACCTT \\
& Reverse: GGACAGATCACAGCCCTCAAT \\
Keap1 & Forward: AACTCGGCAGAATGTTACTACCC \\
& Reverse: CTACGAAAGTCCAGGTCTCTGTCTC \\
NQO1 & Forward: TCAAGAGGAGCAGAAAAAGAACAAG \\
& Reverse: CTGAAAGCAAGCCAGGCAAAC \\
HO-1 & Forward: ATGAGGAACTTTCAGAAGGGTC \\
& Reverse: GTGGGGCATAGACTGGGTT
\end{tabular}

Nrf2, nuclear factor erythroid 2-related factor 2; Keap-1, kelch-like epichlorohydrin-associated protein 1; HO-1, haeme oxygenase-1; NQO1, $\mathrm{NAD}(\mathrm{P}) \mathrm{H}$ dehydrogenase [quinone] 1.

with PrimeScript RT reagent kit (both Takara Biotechnology Co., Ltd., Dalian, China) according to the manufacturer's instructions. Reaction were performed as follows: $37^{\circ} \mathrm{C}$ for $15 \mathrm{~min}, 85^{\circ} \mathrm{C}$ for $5 \mathrm{sec}$. qPCR was then performed using SYBR Premix Ex Taq (Takara Biotechnology Co., Ltd.) using the CFX96 Touch real-time PCR detection system (Bio-Rad Laboratories, Inc., Hercules, CA, USA) following the manufacturers' protocol. Reaction conditions were as follows: $95^{\circ} \mathrm{C}$ for $30 \mathrm{sec}$, followed by 40 cycles of $95^{\circ} \mathrm{C}$ for $5 \mathrm{sec}, 60^{\circ} \mathrm{C}$ for $30 \mathrm{sec}$ and $72^{\circ} \mathrm{C}$ for $30 \mathrm{sec}$. The primers were synthesized by Generay Biotech Co., Ltd. (Shanghai, China). GAPDH was used as an internal control. The primer sequences are listed in Table I. Relative mRNA levels were calculated using the $2^{-\Delta \Delta \mathrm{Cq}}$ method (24).

Western blot analysis. Western blotting was used to determine the protein expression levels of Nrf2, Keap1, HO-1, NQO1 and GAPDH. GAPDH was used as an internal control. Total protein was extracted from the homogenate with radioimmunoprecipitation assay buffer (Beyotime Institute of Biotechnology, Shanghai, China) according to the manufacturer's protocol. Protein concentrations were measured using bicinchoninic acid Protein Assay kit (Nanjing KeyGen Biotech Co., Ltd.). Equal amounts of protein (30 $\mu \mathrm{g} /$ lane) were separated using 10\% SDS-PAGE gels and transferred to polyvinylidene difluoride membranes. Membranes were blocked with $5 \%$ skim milk in TBS with $0.1 \%$ Tween-20 for $1 \mathrm{~h}$ at room temperature, then incubated with anti-Nrf2 (cat.no. ab137550; 1:500; Abcam, Cambridge, UK), anti-Keap1 (cat. no. 7705; Cell Signaling Technology, Inc., Danvers, MA, USA), anti-HO-1 (cat. no. AJ1338b; Abgent, Inc., San Diego, CA, USA), anti-NQO1 (cat. no. ab28947; Abcam; all 1:1,000) or anti-GAPDH (cat. no. KC-5G5; 1:10,000; KangChen BioTech, Co., Ltd., Shanghai, China) antibodies overnight at $4^{\circ} \mathrm{C}$, followed by an incubation with horseradish peroxidase-conjugated goat anti-rabbit antibodies (cat. no. 4050-05; 1:10,000, Southern Biotech, Birmingham, AL, USA) for $1 \mathrm{~h}$ at room temperature. The protein bands were visualized with enhanced chemiluminescence Plus electrochemical luminescence reagent (cat. no. P0018; Beyotime Institute of
Biotechnology). Blots were scanned using the ChemiDoc Imaging system (Bio-Rad Laboratories, Inc.) and then the densitometric analysis of band intensities was performed with Image Lab 2.3 (Bio-Rad Laboratories, Inc.).

Statistical analysis. The data are expressed as mean \pm standard deviation and statistically analysed with SPSS 20.0 (IBM Corp., Armonk, NY, USA). Experiments were repeated $\geq 3$ times. Differences between groups were analysed using one-way analysis of variance followed by Bonferroni post-hoc test. $\mathrm{P}<0.05$ indicated that the difference between groups was statistically significant.

\section{Results}

$B B R$ decreases body weight and decreases liver weight. As shown in Fig. 1, at the end of the experimental period, the body weight at week 6 , liver weight and the liver/body weight ratio in the HFD group were significantly higher compared with the corresponding $\mathrm{NC}$ group (all $\mathrm{P}<0.01$ ). Additionally the body weight at week 4 and 8 in the HFD group were significantly higher compared with the corresponding NC group (both $\mathrm{P}<0.05)$. Compared with the HFD group, the body weight at weeks 6 and 8 , and liver weight in the BBR group were significantly decreased (all $\mathrm{P}<0.01$ ). The liver/body weight ratio in the BBR group was also decreased compared with the HFD group, although not in a significant manner. In addition, rats in the HFD and BBR groups demonstrated decreased food intake, but the differences were not significant.

BBR ameliorates HFD-induced hepatic histopathological changes. As shown in Fig. 2A, histological observations revealed microvesicular steatosis in the livers of the model rats, as demonstrated by excessive small lipid droplets inside the cytoplasm and the swelling of hepatocytes (25). However, there was little evidence of inflammation. This confirmed the successful establishment of the NAFLD model by feeding the rats a high-fat diet for 8 weeks. Rat livers of the NC group exhibited a normal histological structure with no indication of steatosis and inflammation. Compared with the HFD 

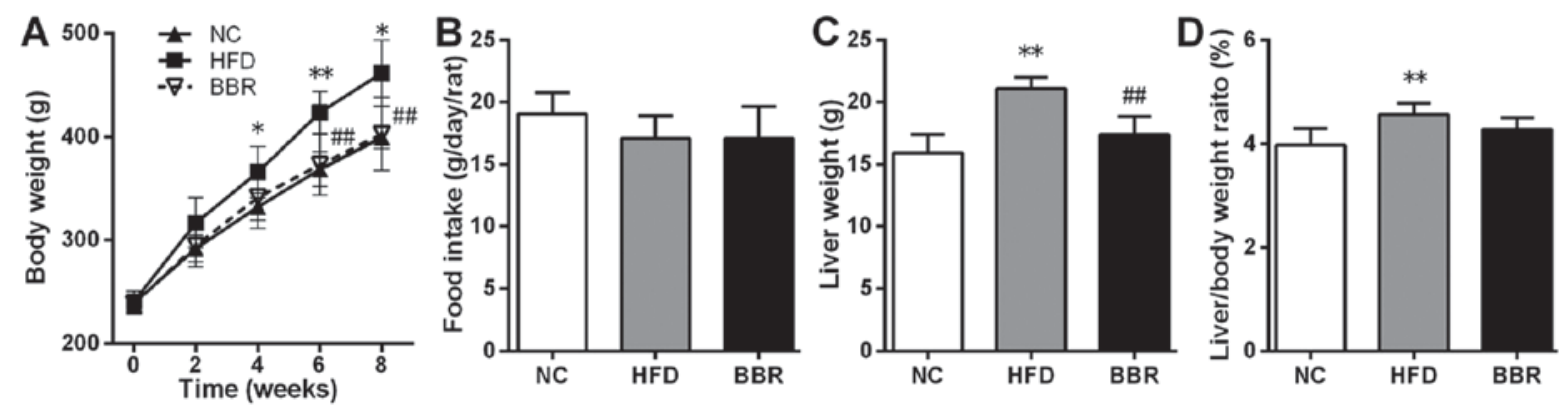

Figure 1. BBR decreases body weight and decreases liver weight. (A) Body weight, (B) food intake, (C) liver weight and (D) liver/body weight ratio. The values are expressed as mean \pm standard deviation. ${ }^{*} \mathrm{P}<0.05$ and ${ }^{* *} \mathrm{P}<0.01$ vs. the $\mathrm{NC}$ group; ${ }^{\#} \mathrm{P}<0.01$ vs. the HFD group. NC, normal control; HFD, high-fat diet; BBR, berberine.
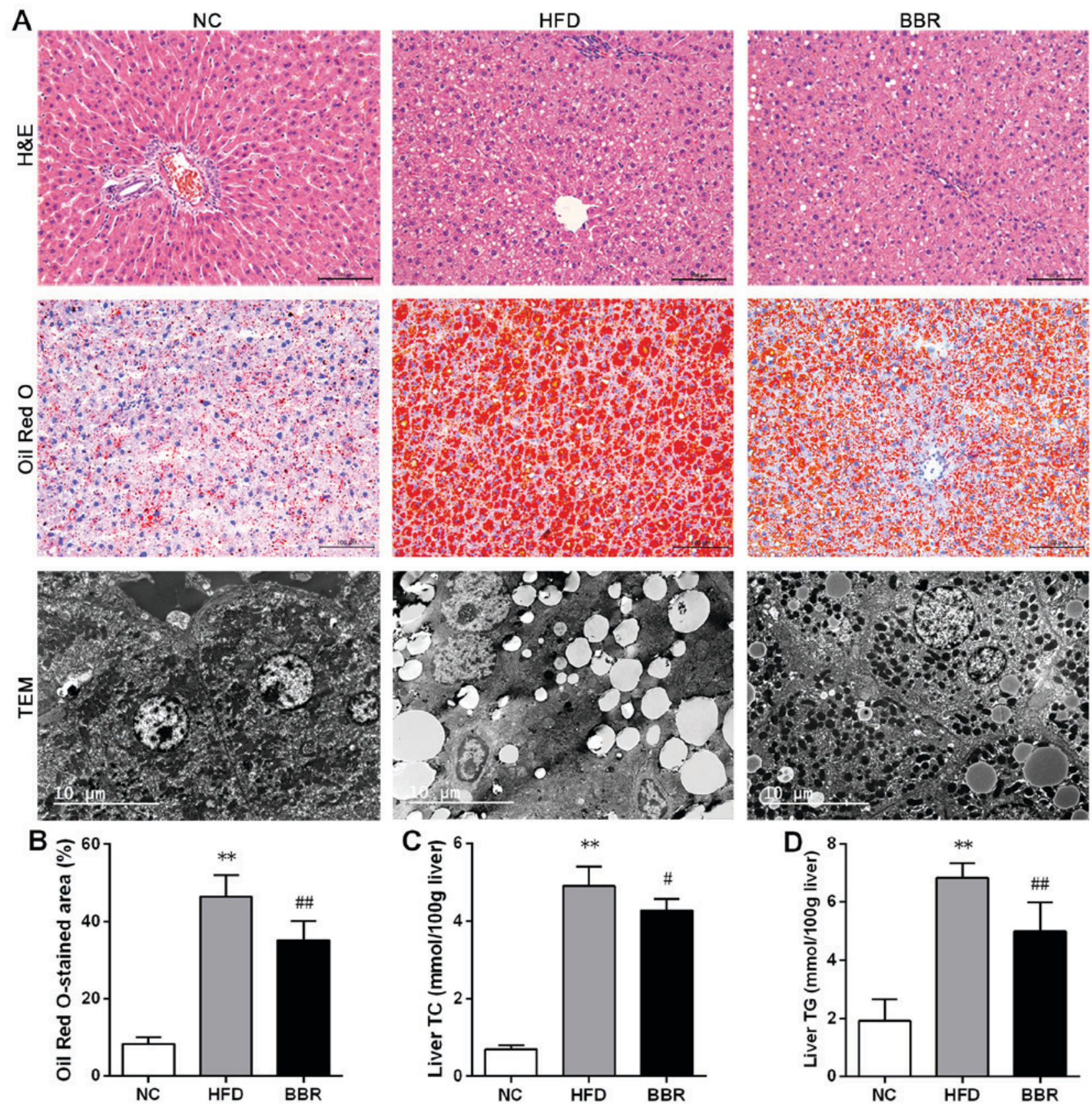

Figure 2. BBR ameliorates HFD-induced hepatic histopathological changes and decreases liver fats. (A) Representative H\&E staining (scale bar, $100 \mu \mathrm{m}$ ), Oil Red O staining (scale bar, $100 \mu \mathrm{m}$ ) and TEM (scale bar, $10 \mu \mathrm{m}$ ) of the liver tissue. (B) The percentage of Oil Red O-stained area was measured. Liver (C) TC and (D) TG levels were assayed. The values are expressed as mean \pm standard deviation. ${ }^{* *} \mathrm{P}<0.01$ vs. the $\mathrm{NC}$ group; ${ }^{*} \mathrm{P}<0.05$ and ${ }^{\# \#} \mathrm{P}<0.01$ vs. the HFD group. NC, normal control; HFD, high-fat diet; BBR, berberine; TC, total cholesterol; TG, triglyceride; TEM, transmission electron microscopy; H\&E, haematoxylin and eosin. 

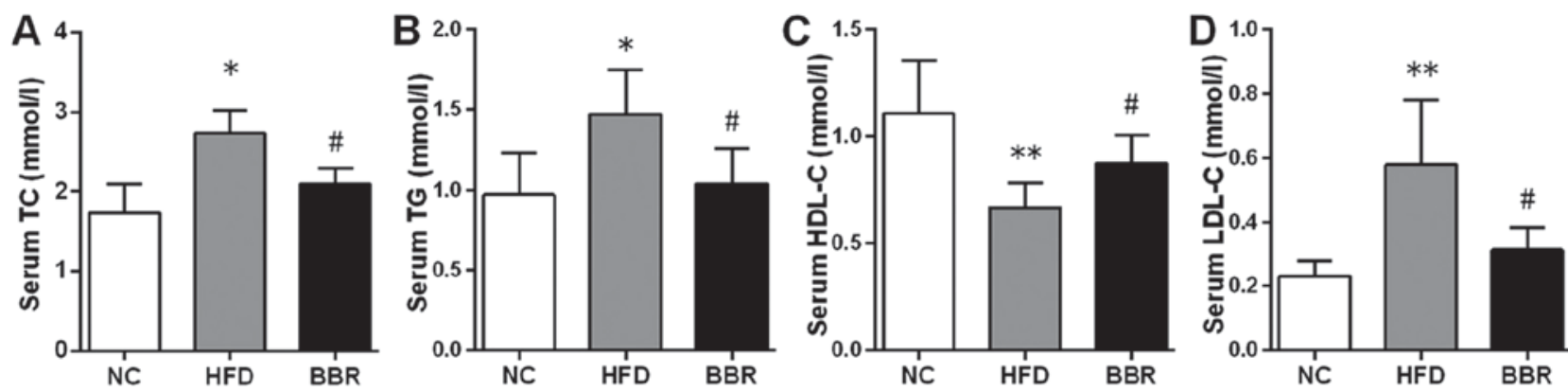

Figure 3. BBR decreases lipid metabolic parameters. Serum levels of (A) TC, (B) TG, (C) HDL-C and (D) LDL-C were assayed in rats. The values are expressed as mean \pm standard deviation. ${ }^{~} \mathrm{P}<0.05$ and ${ }^{* *} \mathrm{P}<0.01$ vs. the $\mathrm{NC}$ group; ${ }^{~} \mathrm{P}<0.05$ and ${ }^{\# \#} \mathrm{P}<0.01$ vs. the HFD group. NC, normal control; HFD, high-fat diet; BBR, berberine; TC, total cholesterol; TG, triglyceride; HDL-C, high-density lipoprotein cholesterol; LDL-C, low-density lipoprotein cholesterol.
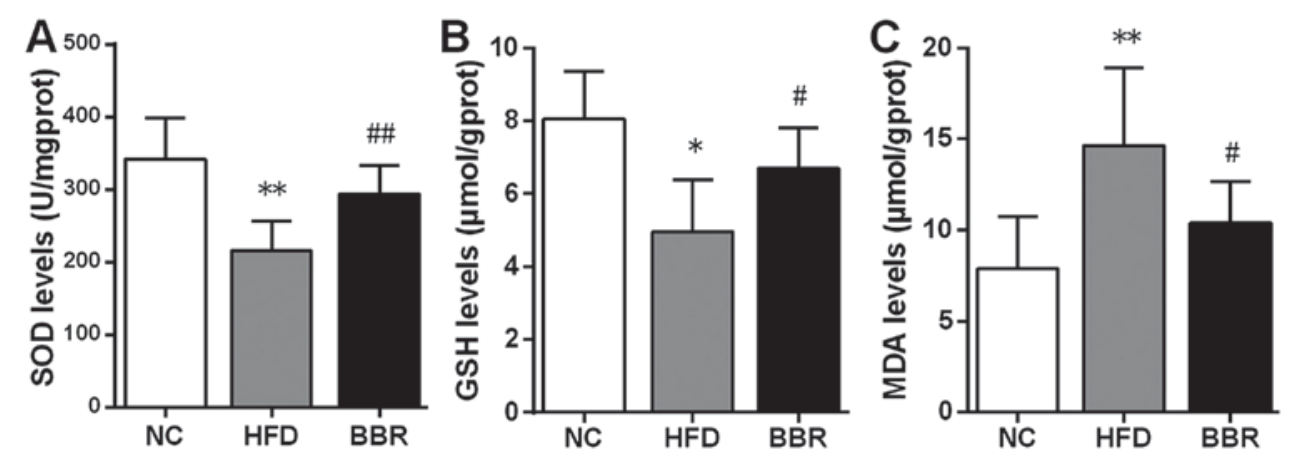

Figure 4. BBR increases SOD and GSH, and decreases MDA. Liver levels of (A) SOD, (B) GSH and (C) MDA were assayed. The values are expressed as mean \pm standard deviation. ${ }^{\mathrm{P}} \mathrm{P}<0.05$ and ${ }^{* * *} \mathrm{P}<0.01$ vs. the $\mathrm{NC}$ group; ${ }^{\#} \mathrm{P}<0.05$ and ${ }^{\# \#} \mathrm{P}<0.01$ vs. the HFD group. NC, normal control; HFD, high-fat diet; BBR, berberine; SOD, superoxide dismutase; GSH, glutathione; MDA, malondialdehyde.

group, the histological structure of rat livers was improved in the BBR group. In addition, the percentage of the Oil Red $\mathrm{O}$-stained area was significantly higher in the HFD group when compared with the NC group ( $\mathrm{P}<0.01$; Fig. $2 \mathrm{~B})$. Transmission electron microscopy demonstrated a decreased number of mitochondria in the HFD group compared with the NC group. In contrast to the HFD group, the intensity of hepatic steatosis was significantly ameliorated in the BBR group, as demonstrated by the decreased percentage of the Oil Red O-stained area $(\mathrm{P}<0.01$; Fig. 2B).

BBR decreases lipid metabolic parameters. To confirm the role of BBR in lipid metabolism, serum and liver lipid profiles were assayed. The histopathological changes revealed that the liver levels of TC and TG were significantly increased in the HFD group compared with the NC group (both $\mathrm{P}<0.01$; Fig. 2C and D). By contrast, the BBR group exhibited significant decreases in the liver levels of TC $(\mathrm{P}<0.05)$ and $\mathrm{TG}$ $(\mathrm{P}<0.01)$ compared with the HFD group. As shown in Fig. 3, compared with the corresponding NC group, serum TC, TG (both $\mathrm{P}<0.05)$ and LDL-C $(\mathrm{P}<0.01)$ levels were significantly increased in the HFD group. The serum HDL-C level was significantly decreased in the HFD group compared with the NC group $(\mathrm{P}<0.01)$. These results suggested the presence of a lipid metabolism disorder in the rats with NAFLD. When BBR was administered, the levels of TC, TG and LDL-C were significantly suppressed while the HDL-C level was significantly increased compared with the HFD group (all $\mathrm{P}<0.05)$.
$B B R$ increases SOD and GSH, and decreases MDA. To evaluate the possible role of BBR in hepatic oxidative stress, SOD, GSH and MDA levels in the liver were assayed. As shown in Fig. 4, rats in the HFD group exhibited significant decreases in SOD $(\mathrm{P}<0.01)$ and GSH $(\mathrm{P}<0.05)$ levels compared with the NC group. By contrast, MDA levels in the HFD group were significantly higher compared with those in the NC group $(\mathrm{P}<0.01)$. In the BBR group, SOD $(\mathrm{P}<0.01)$ and $\mathrm{GSH}$ $(\mathrm{P}<0.05)$ levels were significantly increased, whereas MDA levels $(\mathrm{P}<0.05)$ were significantly reduced compared with those in the HFD group.

BBR increases the expression of genes in the Nrf2/ARE signalling pathway in the liver. To further evaluate the role of the Nrf2/ARE signalling pathway in the development of NAFLD, some key mRNAs and proteins involved in the Nrf2/ARE signalling pathway were measured. As shown in Fig. 5, compared with the NC group, the mRNA levels of Nrf2, HO-1 and NQO1 in the HFD group were significantly increased (all $\mathrm{P}<0.05$ ). Western blot analysis also demonstrated that the expression of HO-1 protein was significantly increased in the HFD group compared with the NC group $(\mathrm{P}<0.01)$, while the expression levels of Nrf2 and NQO1 protein were markedly increased compared with the NC group (Fig. 6). Additionally, BBR administration further increased the mRNA levels of Nrf2, HO-1 and NQO1; they were significantly increased compared with the HFD group (all $\mathrm{P}<0.05$; Fig. 5). Western blot analysis revealed that the protein expression levels of Nrf2, NQO1 (both $\mathrm{P}<0.01)$ and HO-1 $(\mathrm{P}<0.05)$ 
were significantly increased in the BBR group compared with the HFD group (Fig. 6). No significant changes in Keap1 mRNA and protein levels were observed among the groups (Figs. 5 and 6).

\section{Discussion}

In the current study, the authors established a NAFLD rat model by feeding rats a high-fat diet for 8 weeks. The authors demonstrated that H\&E- and Oil Red O-stained liver sections from the model group displayed typical hepatic steatosis, as demonstrated by excessive lipid droplets in the cytoplasm and the swelling of hepatocytes (21). Additionally, serum and liver lipid levels were significantly higher in the model group compared with the normal group. Body weight and liver weight were also significantly increased in the model group. Taken together, these results indicate that rats fed a high-fat diet for 8 weeks can develop the typical signs of hepatic steatosis and lipid metabolism disorder, suggesting that the authors of the current study successfully established a rat model of NAFLD.

Although the pathogenesis of NAFLD remains unclear, the 'two-hit theory' is a widely accepted interpretation of the pathogenic mechanism of NAFLD. The 'first hit' refers to the accumulation of excessive hepatic fat due to insulin resistance, while oxidative stress has been recognized as the 'second hit' in the pathogenesis of NAFLD $(26,27)$. During the 'second hit', oxidative stress caused by excess fat accumulation leads to mitochondrial dysfunction and further liver cell injury, which contributes to the development of NAFLD (8). As important indicators of oxidative stress, antioxidant enzymes, such as SOD, and reactive species, such as MDA, are often used to assess the level of oxidative stress in NAFLD (28). In the current study, the authors observed that rats fed a HFD exhibited a higher MDA level along with lower SOD and GSH levels. The results suggested that oxidative stress could be induced in rat livers after 8 weeks of high-fat feeding.

Over the past decade, a growing body of evidence suggests that activation of the Nrf2/ARE signalling pathway can protect hepatic cells from oxidative stress and prevent the progression of NAFLD $(29,30)$. HO-1 and NQO1 are important antioxidant enzymes regulated by the Nrf2/ARE signalling pathway (9). The results of the current study indicated that the expression of proteins in the Nrf2/ARE signalling pathway was slightly elevated in the livers of the rats high-fat diet-induced NAFLD. This finding indicates that Nrf2 activation could be induced by 8 weeks of high-fat feeding, which is consistent with a previous study (30). Under normal conditions, Nrf2-dependent transcription is repressed by the regulator Keap1 (10). During exposure to oxidative stress, Nrf2 dissociates from Keap1 and is therefore not repressed by Keap1; Nrf2 then translocates into the nucleus where it combines with ARE sequences, activating the downstream transcription of antioxidant enzymes $(11,13)$. The oxidative stress induced by high-fat feeding may activate the Nrf2/ARE signalling pathway, which is important to cellular antioxidant defence (13). A number of studies have demonstrated that, in Nrf2-deficient mice, the inability to adapt to hepatic oxidative stress may hasten the development of NAFLD $(31,32)$. Nevertheless, adaptive Nrf2 activation induced solely by high-fat feeding may not be sufficient to

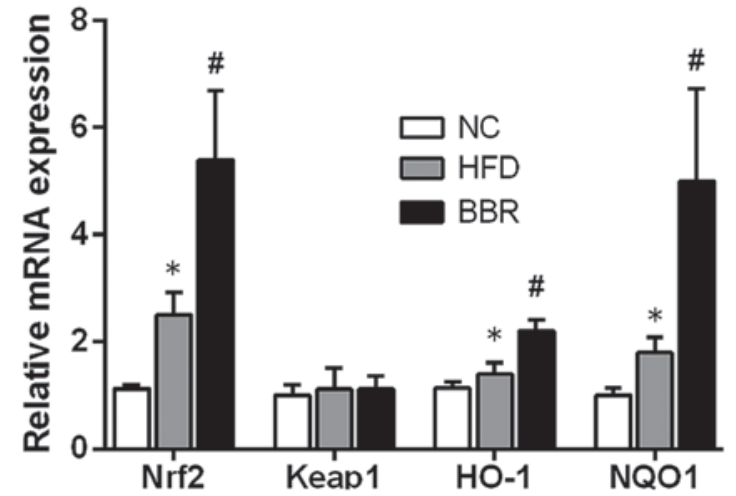

Figure 5. BBR increases the mRNA expression of genes in the Nrf2/ARE signalling pathway in the liver. The values are expressed as mean \pm standard deviation. ${ }^{*} \mathrm{P}<0.05$ vs. the $\mathrm{NC}$ group; ${ }^{\#} \mathrm{P}<0.05$ vs. the HFD group. $\mathrm{NC}$, normal control; HFD, high-fat diet; BBR, berberine; Nrf2, nuclear factor erythroid 2-related factor 2; Keap-1, kelch-like epichlorohydrin-associated protein 1; $\mathrm{HO}-1$, haeme oxygenase-1; NQO1, NAD(P)H dehydrogenase [quinone] 1; ARE, antioxidant response element.

avoid the progression of NAFLD in the absence of an additional Nrf2 activator. Therefore, pharmacological activation of Nrf2 expression has the potential to attenuate the onset of fatty liver disease $(12,31)$. Collectively, these results indicated that Nrf2 serves an important role in inhibiting the progression of NAFLD, and Nrf2 activators have the potential to help prevent and treat this disease.

BBR is a natural compound extracted from the dry roots of Rhizoma coptidis that has been revealed to have beneficial effects on metabolic disorders, such as diabetes mellitus $(33,34)$. Several studies have reported that BBR can reduce body weight, liver weight and serum lipid levels in animal models $(35,36)$. Similarly, the data from the current study demonstrated that BBR exhibits similar beneficial effects in rats with NAFLD induced by a high-fat diet. In the present study, body weight, liver weight and liver lipids in the BBR group were significantly decreased compared with the HFD group. The results of the histopathological evaluation also demonstrated that BBR markedly alleviated hepatic steatosis. These results are in agreement with a previous study our group (21). In brief, these data indicate that BBR successfully slowed down the development of NAFLD in rats through biochemical and histological improvements.

BBR has been reported to attenuate oxidative stress through activation of antioxidative pathways $(18,37)$. Thus, the authors of the current study hypothesized that the Nrf2/ARE signalling pathway may be involved in the mechanisms of the protective effect of BBR in high-fat diet-induced NAFLD. The authors investigated whether BBR can regulate a number of key proteins of the Nrf2/ARE signalling pathway in the liver. The results revealed that the liver levels of the Nrf2, HO-1 and NQO1 proteins in the BBR group were significantly higher when compared with those in the HFD group. These data suggest that BBR administration in rats fed with a high-fat diet could up-regulate Nrf2 expression as well as the expression of some genes and proteins involved in the Nrf2/ARE signalling pathway. As predicted, the results demonstrated that liver MDA content, the product of lipid peroxidation during oxidative stress (8), was decreased by 

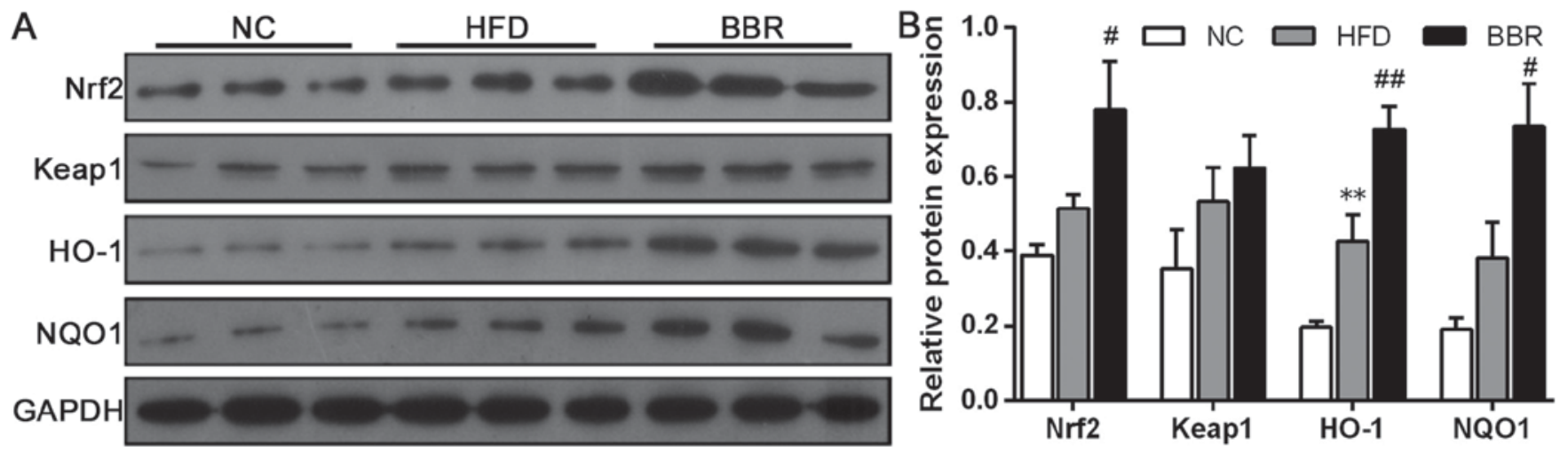

Figure 6. BBR increases the protein expression of genes in the Nrf2/ARE signalling pathway in the liver. (A) Western blot analysis and (B) relative protein expression of Nrf2, Keap1, HO-1 and NQO1. The values are expressed as mean \pm standard deviation. ${ }^{* *} \mathrm{P}<0.01$ vs. the NC group; ${ }^{*} \mathrm{P}<0.05$ and ${ }^{\# \#} \mathrm{P}<0.01$ vs. the HFD group. NC, normal control; HFD, high-fat diet; BBR, berberine; Nrf2, nuclear factor erythroid 2-related factor 2; Keap-1, kelch-like epichlorohydrin-associated protein 1; HO-1, haeme oxygenase-1; NQO1, NAD(P)H dehydrogenase [quinone] 1; ARE, antioxidant response element.

BBR co-administration. Additionally, BBR increased the liver levels of SOD and GSH, which could help to relieve oxidative stress. This effect may have been attributable to the up-regulation of the Nrf2/ARE signalling pathway. In addition, studies have demonstrated that the Nrf2/ARE signalling pathway may be involved in regulation of hepatic lipid metabolism $(11,31)$. A number of pharmacological Nrf2 activators have been reported to reduce liver lipid accumulation and lipogenic gene expression in vivo (38). In the present study, biochemical and histological examination partly confirmed that Nrf2 overexpression following BBR administration reduced lipid content and attenuated hepatic steatosis. Taken together, the findings of the current suggest that BBR can induce the activation of the hepatic Nrf2/ARE signalling pathway, and that this effect may contribute to the amelioration of oxidative stress and its deleterious effects. The Nrf2/ARE signalling pathway may be an important target for BBR in the prevention and treatment of NAFLD. However, the precise mechanisms by which BBR affects the Nrf2/ARE signalling pathway, oxidative stress and lipid metabolism in the development of NAFLD requires further clarification.

In conclusion, the current study demonstrated that BBR may up-regulate the hepatic Nrf2/ARE signalling pathway in rats fed a high-fat diet, and this effect may be associated with the amelioration of oxidative stress. Based on these findings, the authors of the current study concluded that the activation of the Nrf2/ARE signalling pathway may be one of the important mechanisms by which BBR exerts its protective effect against NAFLD.

\section{Acknowledgements}

Not applicable.

\section{Funding}

The present work was supported by Natural Science Foundation of China (grant nos. 81774165 and 81273617) and Traditional Chinese Medicine Bureau of Guangdong Province (grant no. 20152112).

\section{Availability of data and material}

The datasets used and/or analysed during the current study are available from the corresponding author on reasonable request.

\section{Authors' contributions}

QY and YZ designed and organized the study. YD, KT, RC and JZ performed the animal experiments. HN and SL performed the data analysis. YD and KT wrote the manuscript.

\section{Ethics approval and consent to participate}

The present study was approved by the Animal Experimental Ethics Committee of Jinan University (Guangzhou, China).

\section{Patient consent for publication}

Not applicable.

\section{Competing interests}

The authors declare that they have no competing interests.

\section{References}

1. Chalasani N, Younossi Z, Lavine JE, Diehl AM, Brunt EM, Cusi K, Charlton M and Sanyal AJ: The diagnosis and management of non-alcoholic fatty liver disease: Practice Guideline by the American Association for the Study of Liver Diseases, American College of Gastroenterology, and the American Gastroenterological Association. Hepatology 55: 2005-2023, 2012.

2. Than NN and Newsome PN: A concise review of non-alcoholic fatty liver disease. Atherosclerosis 239: 192-202, 2015.

3. Farrell GC and Larter CZ: Nonalcoholic fatty liver disease: From steatosis to cirrhosis. Hepatology 43 (2 Suppl 1): S99-S112, 2006.

4. Musso G, Cassader M and Gambino R: Non-alcoholic steatohepatitis: Emerging molecular targets and therapeutic strategies. Nat Rev Drug Discov 15: 249-274, 2016.

5. Videla LA, Rodrigo R, Araya J and Poniachik J: Insulin resistance and oxidative stress interdependency in non-alcoholic fatty liver disease. Trends Mol Med 12: 555-558, 2006.

6. Friedman SL, Neuschwander-Tetri BA, Rinella M and Sanyal AJ: Mechanisms of NAFLD development and therapeutic strategies. Nat Med 24: 908-922, 2018. 
7. Sakaida I and Okita K: The role of oxidative stress in NASH and fatty liver model. Hepatol Res 33: 128-131, 2005.

8. Rolo AP, Teodoro JS and Palmeira CM: Role of oxidative stress in the pathogenesis of nonalcoholic steatohepatitis. Free Radical Bio Med 52: 59-69, 2012.

9. Kaspar JW, Niture SK and Jaiswal AK: Nrf2:INrf2 (Keap1) signaling in oxidative stress. Free Radical Bio Med 47: 1304-1309, 2009.

10. Vomhof-DeKrey EE and Picklo MJ Sr: The Nrf2-antioxidant response element pathway: A target for regulating energy metabolism. J Nutr Biochem 23: 1201-1206, 2012.

11. Chambel SS, Santos-Goncalves A and Duarte TL: The dual role of Nrf2 in nonalcoholic fatty liver disease: Regulation of antioxidant defenses and hepatic lipid metabolism. Biomed Res Int 2015: 597134, 2015 .

12. Tang W, Jiang YF, Ponnusamy M and Diallo M: Role of Nrf2 in chronic liver disease. World J Gastroentero 20: 13079-13087, 2014

13. Bataille AM and Manautou JE: Nrf2: A potential target for new therapeutics in liver disease. Clin Pharmacol Ther 92: 340-348, 2012.

14. Yin J, Zhang $\mathrm{H}$ and Ye J: Traditional chinese medicine in treatment of metabolic syndrome. Endocr Metab Immune Disord Drug Targets 8: 99-111, 2008.

15. Wang C, Li J, Lv X, Zhang M, Song Y, Chen L and Liu Y Ameliorative effect of berberine on endothelial dysfunction in diabetic rats induced by high-fat diet and streptozotocin. Eur J Pharmacol 620: 131-137, 2009.

16. Kong W, Wei J, Abidi P, Lin M, Inaba S, Li C, Wang Y, Wang Z, $\mathrm{Si} \mathrm{S}$, Pan H, et al: Berberine is a novel cholesterol-lowering drug working through a unique mechanism distinct from statins. Na Med 10: 1344-1351, 2004.

17. Wang Y, Campbell T, Perry B, Beaurepaire C and Qin L: Hypoglycemic and insulin-sensitizing effects of berberine in high-fat diet- and streptozotocin-induced diabetic rats. Metabolism 60: 298-305, 2011.

18. Mo C, Wang L, Zhang J, Numazawa S, Tang H, Tang X, Han X, Li J, Yang M, Wang Z, et al: The crosstalk between Nrf2 and AMPK signal pathways is important for the anti-inflammatory effect of berberine in LPS-stimulated macrophages and endotoxin-shocked mice. Antioxid Redox Sign 20: 574-588, 2014.

19. Liu Y, Zhang L, Song H and Ji G: Update on berberine in nonalcoholic Fatty liver disease. Evid Based Complement Alternat Med 2013: 308134, 2013.

20. Yang J, Ma XJ, Li L, Wang L, Chen YG, Liu J, Luo Y, Zhuang ZJ, Yang WJ, Zang SF and Shi JP: Berberine ameliorates non-alcoholic steatohepatitis in ApoE(-/-) mice. Exp Ther Med 14: 4134-4140, 2017

21. Yang QH, Hu SP, Zhang YP, Xie WN, Li N, Ji GY, Qiao NL, Lin XF, Chen TY and Liu HT: Effect of berberine on expressions of uncoupling protein-2 mRNA and protein in hepatic tissue of non-alcoholic fatty liver disease in rats. Chin J Integr Med 17: 205-211, 2011.

22. Wu D, Wen W, Qi CL, Zhao RX, Lü JH, Zhong CY and Chen YY: Ameliorative effect of berberine on renal damage in rats with diabetes induced by high-fat diet and streptozotocin. Phytomedicine 19: 712-718, 2012.

23. Gomes AP, Duarte FV, Nunes P, Hubbard BP, Teodoro JS Varela AT, Jones JG, Sinclair DA, Palmeira CM and Rolo AP: Berberine protects against high fat diet-induced dysfunction in muscle mitochondria by inducing SIRT1-dependent mitochondrial biogenesis. Biochim Biophys Acta 1822: 185-195, 2012.

24. Livak KJ and Schmittgen TD: Analysis of relative gene expression data using real-time quantitative PCR and the 2(-Delta Delta C(T)) method. Methods 25: 402-408, 2001.
25. Jensen VS, Hvid H, Damgaard J, Nygaard H, Ingvorsen C, Wulff EM, Lykkesfeldt J and Fledelius C: Dietary fat stimulates development of NAFLD more potently than dietary fructose in Sprague-Dawley rats. Diabetol Metab Syndr 10: 4, 2018.

26. Malaguarnera M, Di Rosa M, Nicoletti F and Malaguarnera L: Molecular mechanisms involved in NAFLD progression. J Mol Med (Berl) 87: 679-695, 2009.

27. Day CP and James OF: Steatohepatitis: A tale of two 'hits'? Gastroenterology 114: 842-845, 1998.

28. Zhu R, Wang Y, Zhang L and Guo Q: Oxidative stress and liver disease. Hepatol Res 42: 741-749, 2012.

29. Tanaka Y, Aleksunes LM, Yeager RL, Gyamfi MA, Esterly N, Guo GL and Klaassen CD: NF-E2-related factor 2 inhibits lipid accumulation and oxidative stress in mice fed a high-fat diet. J Pharmacol Exp Ther 325: 655-664, 2008.

30. Xu W, Shao L, Zhou C, Wang H and Guo J: Upregulation of Nrf2 Expression in Non-Alcoholic Fatty Liver and Steatohepatitis. Hepatogastroenterology 58 : 2077-2080, 2011.

31. Okada K, Warabi E, Sugimoto H,Horie M, Gotoh N, Tokushige K, Hashimoto E, Utsunomiya H, Takahashi H, Ishii T, et al: Deletion of Nrf2 leads to rapid progression of steatohepatitis in mice fed atherogenic plus high-fat diet. J Gastroenterol 48: 620-632, 2013.

32. Meakin PJ, Chowdhry S, Sharma RS, Ashford FB, Walsh SV, McCrimmon RJ, Dinkova-Kostova AT, Dillon JF, Hayes JD and Ashford ML: Susceptibility of Nrf2-Null mice to steatohepatitis and cirrhosis upon consumption of a high-fat diet is associated with oxidative stress, perturbation of the unfolded protein response, and disturbance in the expression of metabolic enzymes but not with insulin resistance. Mol Cell Biol 34: 3305-3320, 2014.

33. Li Z, Geng Y, Jiang J and Kong W: Antioxidant and anti-inflammatory activities of berberine in the treatment of diabetes mellitus. Evid-Based Compl Alt 2014: 289264, 2014.

34. Yin J, Xing $\mathrm{H}$ and Ye J: Efficacy of berberine in patients with type 2 diabetes mellitus. Metabolism 57: 712-717, 2008

35. Chang X, Yan H, Fei J, Jiang M, Zhu H, Lu D and Gao X: Berberine reduces methylation of the MTTP promoter and alleviates fatty liver induced by a high-fat diet in rats. J Lipid Res 51: 2504-2515, 2010.

36. Zhou J, Zhou S, Tang J, Zhang K, Guang L, Huang Y, Xu Y, Ying Y, Zhang L and Li D: Protective effect of berberine on beta cells in streptozotocin- and high-carbohydrate/high-fat diet-induced diabetic rats. Eur J Pharmacol 606: 262-268, 2009.

37. Hsu YY, Chen CS, Wu SN, Jong YJ and Lo YC: Berberine activates Nrf2 nuclear translocation and protects against oxidative damage via a phosphatidylinositol 3-kinase/Akt-dependent mechanism in NSC34 motor neuron-like cells. Eur J Pharm Sci 46: 415-425, 2012

38. Yates MS, Tran QT, Dolan PM, Osburn WO, Shin S, McCulloch CC, Silkworth JB, Taguchi K, Yamamoto M, Williams CR, et al: Genetic versus chemoprotective activation of Nrf2 signaling: Overlapping yet distinct gene expression profiles between Keapl knockout and triterpenoid-treated mice. Carcinogenesis 30: 1024-1031, 2009.

This work is licensed under a Creative Commons Attribution-NonCommercial-NoDerivatives 4.0 International (CC BY-NC-ND 4.0) License. 